\title{
Single-cell analysis reveals congruence between kidney organoids and human fetal kidney
}

\author{
Alexander N. Combes ${ }^{1,2^{*}}$, Luke Zappia ${ }^{2,3+}$, Pei Xuan $\mathrm{Er}^{2}$, Alicia Oshlack ${ }^{2,3}$ and Melissa H. Little ${ }^{1,2,3,4^{*}}$ (D)
}

\begin{abstract}
Background: Human kidney organoids hold promise for studying development, disease modelling and drug screening. However, the utility of stem cell-derived kidney tissues will depend on how faithfully these replicate normal fetal development at the level of cellular identity and complexity.
\end{abstract}

Methods: Here, we present an integrated analysis of single cell datasets from human kidney organoids and human fetal kidney to assess similarities and differences between the component cell types.

Results: Clusters in the combined dataset contained cells from both organoid and fetal kidney with transcriptional congruence for key stromal, endothelial and nephron cell type-specific markers. Organoid enriched neural, glial and muscle progenitor populations were also evident. Major transcriptional differences between organoid and human tissue were likely related to technical artefacts. Cell type-specific comparisons revealed differences in stromal, endothelial and nephron progenitor cell types including expression of WNT2B in the human fetal kidney stroma.

Conclusions: This study supports the fidelity of kidney organoids as models of the developing kidney and affirms their potential in disease modelling and drug screening.

Keywords: Single-cell RNA sequencing, Human kidney organoids, Stem cell-derived models, Induced pluripotent cells, Organoids

\section{Background}

Knowledge of developmental programs can be used to direct the differentiation of human-induced pluripotent stem cells towards a desired cell fate. Such approaches have successfully generated models of human intestinal epithelium, brain and ear, in each instance forming multicellular self-organising structures termed organoids by mimicking conditions that regulate development of the same tissues during embryogenesis [1]. Similarly, protocols for the generation of human kidney cell types have been developed by ourselves [2,3] and others [4-8]. Such protocols raise the exciting prospect of disease modelling, toxicity and drug screening in vitro and open new opportunities for regenerative medicine. However, the value of

\footnotetext{
* Correspondence: alexander.combes@unimelb.edu.au; melissa.little@mcri.edu.au

${ }^{\dagger}$ Alexander N. Combes and Luke Zappia contributed equally to this work. ${ }^{1}$ Department of Anatomy \& Neuroscience, University of Melbourne, Melbourne, VIC, Australia

Full list of author information is available at the end of the article
}

stem cell-derived kidney tissue will depend on how faithfully it represents human renal tissue, the degree to which the component cell types mature and the absence of confounding cell types within such cultures.

Component cell types present within kidney organoids have primarily been defined by detecting established markers of murine renal cell types by immunofluorescence. This has identified cell types with similarity to endothelial cells $\left(\mathrm{CD} 31^{+}\right)$, stroma $\left(\mathrm{MEIS1}^{+}\right)$, nephron progenitor cells $\left(\mathrm{SIX}^{+}, \mathrm{HOXD}_{1}{ }^{+}, \mathrm{WT}^{+}, \mathrm{PAX}^{+}\right)$, and epithelial structures with markers of the ureteric epithelium $\left(\mathrm{PAX}^{+}, \mathrm{GATA}^{+}, \mathrm{CDH}^{+}\right)$, renal vesicle $\left(\mathrm{JAG}^{+}\right)$, distal tubule $\left(\mathrm{CDH}^{+}, \mathrm{GATA}^{-}\right)$, loop of Henle $\left(\mathrm{UMOD}^{+}\right.$, $\left.\mathrm{CDH}^{+}\right)$, proximal tubule $\left(\mathrm{LTL}^{+} \mathrm{CDH1}^{-}, \mathrm{CUBN}^{+}\right)$and podocytes $\left(\mathrm{NPHS}^{+}\right)[2,3]$. However, the extent to which cell identity is conserved beyond these key markers is unclear. Indeed, as these markers were selected based on an understanding of mouse kidney development, they are not definitive evidence of an appropriate human cell type.

(C) The Author(s). 2019 Open Access This article is distributed under the terms of the Creative Commons Attribution 4.0 International License (http://creativecommons.org/licenses/by/4.0/), which permits unrestricted use, distribution, and 
Similarly, there can be variation between kidney organoid experiments and differentiation protocols as well as between starting cell lines. Finally, it is likely that the differentiation is imperfect, resulting in variable populations of off-target cell types.

We recently profiled gene expression in over 8000 single cells from two batches of human pluripotent stem cell-derived kidney organoids as part of an analysis of experimental variation in our organoid protocol [9]. Here, we independently analyse that data to characterise the cellular composition of kidney organoids, extending the expression profiles of expected endothelial and nephron cell types, and identifying subpopulations expressing stromal markers, and off-target glial, neural and muscle progenitors. Despite expression of recognisable renal markers, there remains the possibility of broader underlying differences between kidney organoid cell types and equivalent populations in the developing human kidney. To directly compare these tissues, we integrated single-cell RNA sequencing (scRNA-seq) data from kidney organoids with publicly available human fetal kidney (hFK) data [10]. Our analysis shows that clusters corresponding to stromal, nephron and endothelial populations included cells from both organoid and hFK origin. We further identified populations that were specific to each dataset. Substantial conservation of cell type-specific markers between cells from hFK and organoids was observed whereas differences between tissues were obscured by a strong signature that was found to be technical rather than biological. After identifying and removing genes associated with that signature, cell type-specific differences in stromal, endothelial and nephron progenitor cells emerged, indicating avenues for improving organoid cell types. This analysis extends our understanding of the cellular composition of human kidney organoids and demonstrates that kidney organoids reproduce several cell types found in the developing human kidney.

\section{Methods}

This study aimed to characterise the cellular composition of human kidney organoids and compare organoid cell types to equivalent cell types in the developing human kidney. Two batches of organoids were produced and profiled. The resulting scRNA-seq dataset was then integrated with and compared to publicly available human fetal kidney scRNA-seq data [10] as outlined below.

\section{Organoid differentiation and single-cell experiments}

Kidney organoids were made and stained according to our published protocol [11] from human-induced pluripotent stem cell line CRL1502 [12]. Three organoid samples were differentiated to day 25 ( 7 days of monolayer culture plus 18 days as a 3D aggregate). A further organoid was differentiated to day 25 in a second independent experiment.
Organoids were dissociated and run on 10x Chromium Single Cell Chips as previously described [9]. Additional organoids were differentiated to day 24 for validation of glial and muscle progenitor populations. Immunofluorescence was performed according to our published protocol using antibodies detailed in that report [11]. Additional antibodies to FABP7 (Abcam Rabbit anti-BLBP \#ab32423) and MYOG (Abcam Mouse anti MYOG \#ab1835) were used at 1:300.

\section{Pre-processing}

The Cell Ranger pipeline (v1.3.1 10X Genomics) was used to perform sample demultiplexing, barcode processing and single-cell gene counting. Briefly, samples were demultiplexed to produce a pair of FASTQ files for each sample. Reads containing sequence information were aligned to the GRCh38 reference genome provided with Cell Ranger (v1.2.0). Cell barcodes were filtered to remove empty droplets, and PCR duplicates were removed by selecting unique combinations of cell barcodes, unique molecular identifiers and gene ids with the final results being a gene expression matrix that was used for further analysis. The three samples in the first batch of organoids were aggregated using Cell Ranger with no normalisation and treated as a single dataset.

\section{Quality control}

The R statistical programming language (v3.5.0) [13] was used for further analysis. Count data for each experiment was read into $\mathrm{R}$ and used to construct a SingleCellExperiment object (v1.2.0) [14]. Gene annotation information was added from BioMart [15] using the biomaRt package (v2.36.1) [16], and cells were assigned cell cycle scores using the cyclone [17] function in the scran package (v1.8.2) [18].

The scater package (v1.8.2) [19] was used to produce a series of diagnostic quality control plots. Cells with a high number of expressed genes (indicating potential doublets) were removed, as were cells with a high percentage of counts assigned to mitochondrial or ribosomal genes, or with low expression of the housekeeping genes GAPDH and ACTB.

Genes that had less than two total counts across a dataset, or were expressed in less than two individual cells, were removed. We also removed genes without an annotated HGNC symbol.

Following quality control, the first organoid dataset consisted of 6649 cells and 18,386 genes with a median of 2738 genes expressed per a cell, the fourth organoid had 1288 cells and 16,885 genes with a median of 3248 genes expressed per cell and the human developing kidney dataset [10] had 3178 cells and 16,166 genes with a median of 1509.5 genes expressed per cell. 


\section{Clustering analysis Organoids}

The two organoid datasets were integrated using the alignment method in the Seurat package (v2.3.1) [20, 21]. Briefly, highly variable genes were identified in each dataset and those that were present in both datasets (1156 genes) were selected. Canonical correlation analysis $[22,23]$ was then performed using the selected genes and 25 dimensions that represent the majority of variation were selected. The final step used dynamic time warping [24] to align the datasets in the selected subspace.

To perform clustering, Seurat constructs a shared nearest neighbour graph of cells in the aligned subspace and uses the Louvain modularity optimisation [25] to assign cells to clusters. The number of clusters produced using this method is controlled by a resolution parameter with higher values giving more clusters. We performed clustering over a range of resolutions from 0 to 1 in steps of 0.1 and used the Clustree package (v0.2.2.9000) to produce clustering trees [26] showing the expression of known marker genes to select the appropriate resolution to use. We chose to use a resolution of 0.6 which produced 13 clusters.

Marker genes for each cluster were detected by testing for differential expression between cells in one cluster and all other cells using a Wilcoxon rank sum test [27]. To reduce processing time, only genes that were expressed in at least $10 \%$ of cells in one of these groups were tested. We chose the $10 \%$ cutoff over the default of $25 \%$ in order to return results for more genes. To identify conserved marker genes, a similar process was performed on each dataset separately and the results combined using the maximum $p$ value method. We also tested for within cluster differential expression to identify differences between cells of the same type in different datasets.

Based on identified marker genes, we determined clusters 2 and 9 represented the nephron lineage. The 1125 cells in these clusters were re-clustered at a resolution of 0.5 resulting in 5 clusters.

We also performed pseudotime trajectory analysis on the nephron cells using Monocle (v2.8.0) [28, 29]. The intersection of the top 100 genes with the greatest absolute fold change for each nephron cluster was selected for this analysis, giving a set of 455 genes used to order the cells.

\section{Combined}

The combined organoid and human fetal kidney analysis used the procedure described for the organoid-only analysis but with slightly different parameters. We identified 1368 variable genes present in all three datasets and selected the first 20 canonical correlation dimensions. For clustering, we chose a resolution of 0.5 which produced 16 clusters. Clusters $6,7,10$ and 15 were determined to be the nephron lineage and these 1964 cells were reclustered at a resolution of 0.6 producing 8 clusters.

We also performed differential expression testing between the two datasets as a whole, which was used to identify a signature of 374 genes that represent the main differences between them. To identify cell type-specific differences between organoid and human fetal kidney, we performed differential expression testing between cells within a cluster and removed genes found in the overall differential expression signature. Cluster 7 in the combined nephron analysis was identified as a human fetal kidney specific podocyte cluster. To investigate the differences between these cells and other podocytes, we compared gene expression in this cluster to the general podocyte cluster (CNO).

\section{Visualisation and presentation}

Figures shown here were produced using functions in the Seurat, Monocle and Clustree packages. Additional plots and customisations were created using the ggplot2 (v3.0.0) [30] and cowplot (v0.9.3) [31] packages. The analysis project was managed using the workflowr (v1.1.1) (50) package which was also used to produce the publicly available website displaying the analysis code, results and output.

\section{Results}

Segmented epithelial nephrons, stroma and endothelial cells have been identified within human kidney organoids by correlating tissue morphology with established markers of equivalent cell types in mouse. However, several markers unique to a cell type within a developing kidney are also expressed in divergent cell types throughout the embryo. Therefore, it is unclear how closely kidney organoid cell types align with cells specified in an in vivo environment and characterising cell types derived from pluripotent cells in vitro must rely on combinations of markers unique to the cell type of interest. We employed scRNA-seq to analyse the cellular composition of kidney organoids, aiming to obtain profiles of the constituent cell types to complement and extend our prior characterisation by immunofluorescence.

\section{Defining the cellular composition of human kidney organoids using high-throughput single-cell RNA sequencing}

Our analysis of the sources of variation between kidney organoid differentiations included scRNA-seq data from over 8000 cells isolated from day 25 kidney organoids, identifying populations with similarity to nephron progenitor, nephron tubule, podocyte, endothelium and three stromal populations, as well as a small immune-like cluster and off-target neural populations [9]. That study highlighted experimental batch as the strongest contributor to transcriptional variation with evidence for shifts in 
relative maturation as a major confounder. In this study, we perform an independent analysis of that data ("Methods"). Each aspect of the current analysis is documented in a website at http://oshlacklab.com/combes-organoid-paper/, including further information and figures detailing quality control, clustering, marker and differential expression analysis [32]. We generated a combined dataset representing 7937 cells by integrating cells from four organoids across two batches (Additional file 1) and performed clustering over a range of resolutions $(0-1$ in 0.1 increments) using Seurat [20]. The Clustree package [26] was used to visualise how clusters changed with increasing resolution and select an appropriate resolution to use (Additional file 1). Thirteen organoid $(\mathrm{O})$ clusters were identified at resolution 0.6 including clusters representing stromal, nephron, endothelial cells and cell types not usually present in a developing mouse kidney (Fig. 1a). Cluster identity was established by comparison of cluster marker genes to established markers of known murine and human renal cell types [10, 33-35]

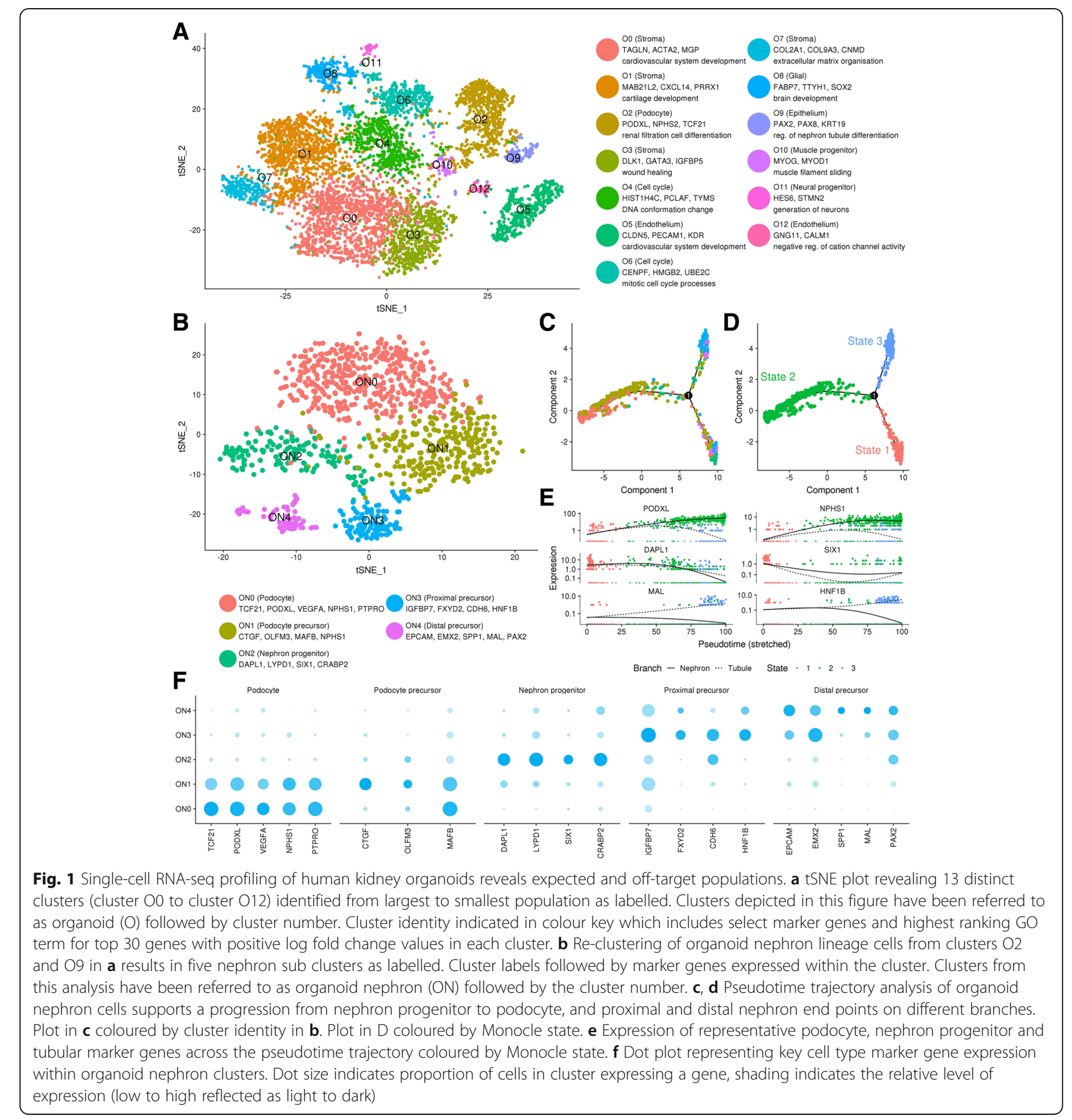


and by gene ontology (GO) analysis through ToppFun [36] (Additional file 2). The number and identity of clusters was largely consistent with our previous independent analysis [9], and the readily identifiable renal populations are dealt with below. Less identifiable and potentially off-target populations included a small neural population marked by ELAVL3 and ELAVL4, and a more substantial population expressing glial markers including FABP7. An additional off-target population of muscle progenitors expressing MYOD1, MYOG and PITX2 was detected for the first time (Fig. 1a). While smooth muscle cells play an important role in expelling renal filtrate from the kidney to the bladder, the presence of cells expressing muscle progenitor markers might suggest a developmentally off-target population. Immunofluorescence for MYOG identified scattered muscle progenitors in small numbers on the surface of the organoid (Additional file 1). FABP7-expressing presumptive glial cells were also present at a low frequency in the organoid stroma (Additional file 1). The presence of a detectable cluster for what appeared to be a relatively rare and peripheral cell population may result from differential dissociation of these populations during preparation of material for single-cell analysis. We were unable to detect cells expressing neural markers ELAVL3, ELAVL4 or NCAM1 with antibodies to these proteins. This neural cluster corresponds to a small proportion of the overall cells (0.9\%). A lack of detectable cells within organoids when using antibody markers may reflect the rarity of the population or variations in the presence of this population between organoid differentiations.

The developing kidney is known to have distinct stromal subpopulations. While four stromal populations were evident within kidney organoids, unifying markers of these populations (e.g. MEIS1, PDGFRA, COL3A1) are expressed in stromal tissues throughout the mouse embryo and hence are not sufficient to identify these clusters as cell types specific to the developing kidney. One of these clusters $(\mathrm{O} 3)$ is marked by GATA3 and is evident by immunofluorescence (Additional file 1). GATA3 is known to be expressed in mesangial cells within the glomerulus and in vascular associated cells [37]. In organoids, we observe expression of GATA3 in cells scattered throughout the stromal compartment.

\section{Analysis of organoid nephron cell types reveals a persistent nephron progenitor-like population}

To interrogate the nephron and epithelial populations further, we re-clustered cells from clusters $\mathrm{O} 2$ (podocyte) and $\mathrm{O} 9$ (nephron epithelium) at multiple resolutions. We selected resolution 0.5 for analysis as this was the first resolution to split the epithelial cluster (O9) into subpopulations [32]. This re-clustering produced five organoid nephron $(\mathrm{ON})$ clusters with similarity to nephron progenitor cells (ON2 marked by DAPL1, LYPD1, SIX1, CRABP2), podocyte precursors as seen in the proximal early nephron (ON1 CTGF, OLFM3, MAFB, NPHS1, low levels of LHX1 and $P A X 8$ ), podocytes (ON0 marked by TCF21, PODXL, $V E G F A, W T 1)$, proximal tubule precursors (ON3 IGFBP7, FXYD2, CDH6, HNF1B), and cluster expressing markers common to the ureteric epithelium and distal nephron (EPCAM, EMX2, SPP1, MAL and PAX2 (ON4)) (Fig. 1b, Additional file 3 ). Though all of these cell types expressed markers conserved in mice, the top differentially expressed markers of nephron progenitor and proximal early nephron cells were recently defined markers specific to human kidney cell types $[38,39]$. The $E P C A M^{+}$ $P A X 2^{+}$cluster $\mathrm{ON} 4$ also expressed markers enriched in the distal tubule such as DEFB1 and TMEM52B. While we could find no markers previously identified as unique to the mouse ureteric epithelium, immunofluorescence for $\mathrm{CDH}^{+} \mathrm{GATA}^{+}$structures previously defined as presumptive ureteric epithelium revealed the presence of these structures at a low frequency in the organoids profiled for scRNA-seq (Additional file 1). The reduced presence of this presumptive ureteric epithelium likely reflects the use of a 'posteriorised' differentiation using an initial culture (4 days of $8 \mu \mathrm{M}$ CHIR). As previously described [3], this substantially reduces the presence of this epithelial segment. In addition, this cell type may be selectively lost due to inefficient dissociation. Some markers of maturing proximal $(S L C 3 A 1, C U B N)$ and distal tubule/loop of Henle $(U M O D)$ were not detected in this data, despite being identified in previous bulk transcriptional profiling and immunofluorescence of older organoids (day 25) $[40,41]$. Again, this may represent relative depletion of epithelial cell types during dissociation.

While our current kidney organoid protocol shows evidence of nephron formation and segmentation, previous bulk RNA-seq analysis suggested a peak of nephron progenitor marker expression early (days 14 to 17 ) followed by a significant decline with time [3]. We identify a cluster expressing nephron progenitor markers in these late (day 25) human kidney organoids (ON2). In the developing mouse kidney, nephron progenitors are thought to be dependent on supporting signals from the neighbouring ureteric tip and surrounding stroma, cumulatively referred to as the nephrogenic niche. This data suggests a nephron progenitor-like population is maintained in these kidney organoids apparently independent of the ureteric tip. Organoid stromal populations may be providing some supporting signals for these cells that likely represent progenitors that have failed to form a nephron.

We next used Monocle $[28,29]$ to order these organoid nephron cells along a continuous pseudotime trajectory and visualised cell types by cluster (Fig. 1c) and by Monocle-determined cell state (Fig. 1d). Based on mouse lineage tracing [42] and single-cell trajectories in human [38], we would expect a nephron progenitor state 
to diverge into separate trajectories for podocyte, distal and proximal tubule. This expected trajectory is maintained in the organoid cell types with a nephron progenitor-enriched population that diverges into separate trajectories for podocyte and nephron tubule cell types. The proximal early nephron cluster (ON1) leads to a more mature podocyte endpoint (ONO) as predicted from the marker analysis. The proximal and distal nephron precursor clusters occupy a separate trajectory, analogous to similar results in the developing human kidney [38]. Gene expression along the pseudotime trajectory and across cell clusters shows expected segregation of key cell type markers (Fig. 1e, f). This analysis suggests that organoid nephron differentiation proceeds in a manner analogous to that which occurs in an in vivo environment.

Overall, this analysis of kidney organoid scRNA-seq data supports the presence of previously characterised nephron and endothelial cell populations while increasing confidence in each by identifying robust sets of co-expressed cell type-specific markers. New insight revealed by this approach includes identification of a persistent nephron progenitor-like population, stromal subpopulations, and off-target glial, neural and muscle progenitor populations.

\section{Comparison to human developing kidney reveals conserved endothelial, nephron and stromal populations} Recent transcriptional comparisons between kidney development in mouse and human have identified human-specific cell type markers [10, 39], many of which were expressed in the human kidney organoid nephron cell types detailed above. Despite expression of these key markers, the possibility of broader underlying differences between nephron cell types in kidney organoids and the developing human kidney remained. Likewise, the extent of similarity between organoid and human fetal kidney stromal cell types was unclear. To facilitate direct comparison between organoid and human fetal kidneys, we combined our organoid single cell data with published hFK single cell data [10] using the Seurat alignment method based on canonical correlation analysis and dynamic time warping [20]. The hFK cells were obtained at 16 weeks of development, a period of active branching morphogenesis and nephron formation [10]. The combined dataset represented expression information for 18,812 genes within 11,115 cells. We again clustered at multiple resolutions and used a clustering tree to select a resolution of 0.5 for analysis. This resulted in 16 combined (C) clusters including five stromal clusters $(\mathrm{C} 0, \mathrm{C} 1, \mathrm{C} 2, \mathrm{C} 3, \mathrm{C} 9)$, an endothelial cluster $(\mathrm{C} 4)$, four nephron lineage clusters (C6, C7, C10, C15), two clusters related to cell cycle (C5, C8), glial (C11) and neural (C14) clusters, an immune cluster (C12), and a blood cell cluster (C13) (Fig. 2a, b; Additional file 4). Organoid cells were grouped in similar clusters in the combined data set compared to the previous organoid-only analysis (Fig. 2c) suggesting that we have identified the same populations in both the organoid-only and combined analyses.

We anticipated differences in the cell types present in fetal kidney and kidney organoids as the hFK data represented cells dissociated from the nephrogenic zone of the outer cortex rather than the whole organ. Previous analysis showed the hFK dataset contains cortical stroma, early nephron, vascular, blood and immune cell types, but it does not contain ureteric epithelium, mature nephrons or medullary stromal populations [10]. Conversely, kidney organoids contain populations designated as off-target given they were not intentional outcomes of the kidney organoid differentiation protocol. With these caveats in mind, we assessed the similarity between cell types in these datasets by determining the contributions of kidney organoid and hFK cells to each cluster (Fig. 2d), and the proportions of general cell types between the samples (Fig. 2e). This showed substantial contributions of both organoid and hFK cells to stromal, endothelial and nephron clusters. Importantly, this combined analysis allowed us to allocate small numbers of cells to clusters not previously identified in the individual tissue analysis such as a small group of organoid cells expressing immune response genes within $\mathrm{C} 12$, and fetal kidney cells expressing glial and neural markers within $\mathrm{C} 11$ and $\mathrm{C} 14$. Neural cells were not identified in the original analysis of this hFK data [10] but these cells have been found at low frequency in other datasets from the human fetal kidney [43].

\section{Top tissue type differences relate to cellular stress in the fetal kidney data and growth in organoids}

Co-clustering the organoid and hFK data provided a means to analyse conserved and differential transcriptional profiles within cell types. Initial differential expression analysis between hFK and organoid cell types within each cluster revealed recurring sets of genes that confounded further analysis. We performed differential expression analysis between all cells in the organoid and hFK samples to formally identify genes that were enriched in either dataset (Fig. 2f). GO analysis of genes upregulated in the hFK cells returned terms including 'response to unfolded protein,' 'regulation of programmed cell death' and 'response to temperature stimulus', consistent with heat shock and cellular stress. GO terms associated with organoid enriched genes relate to an abundance of ribosomal proteins, including 'translation initiation' and 'ribosome biogenesis', consistent with enhanced growth (Additional file 5). This heat shock or stress signature in the hFK data is a potential side effect of cell dissociation [44] but could also reflect the fact that the fetal material was acquired at termination and 

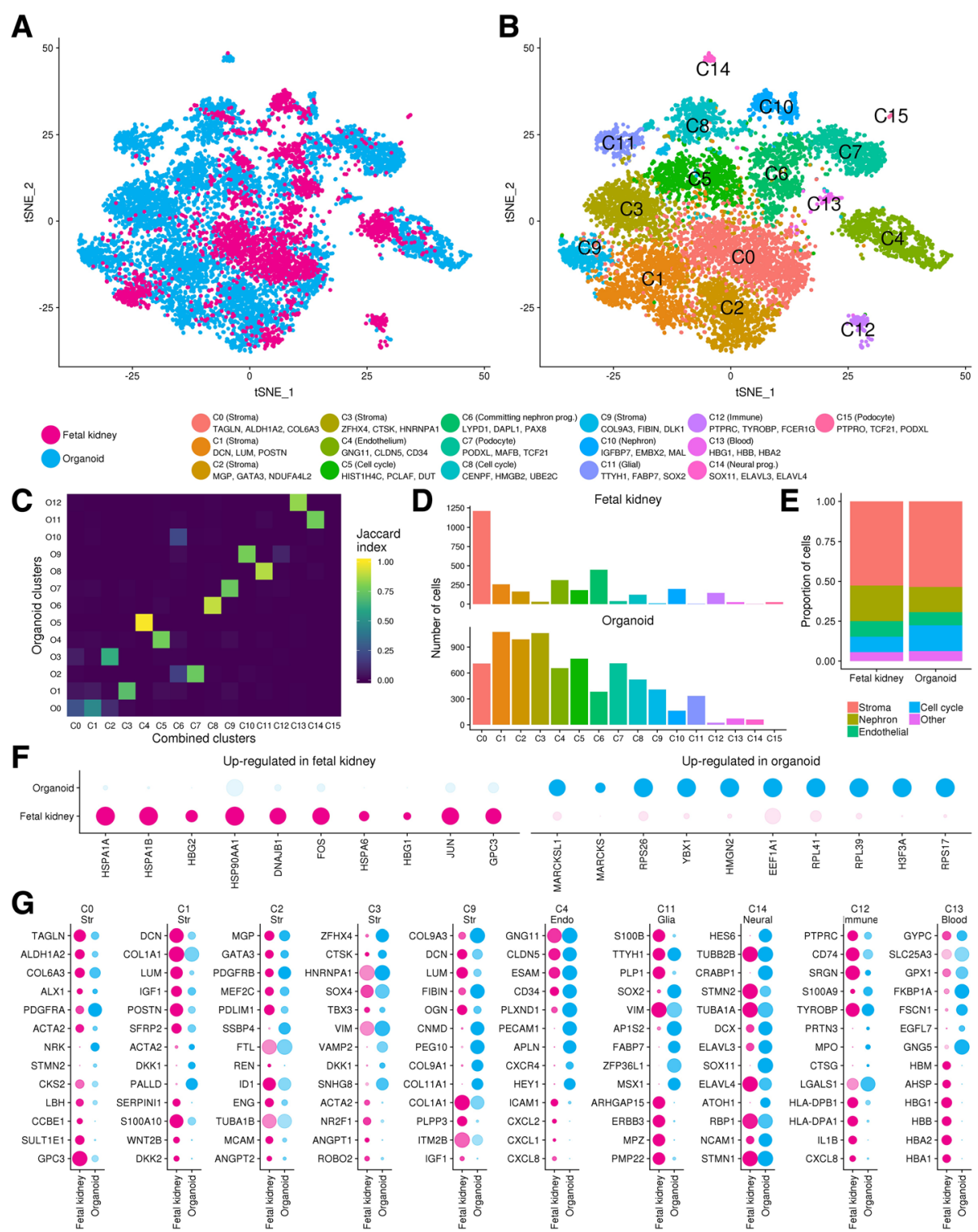

Fig. 2 Integration and comparison of kidney organoids and human fetal kidney scRNA-seq. a tSNE plot of combined organoid and hFK data coloured by sample type. b tSNE plot revealing 16 'combined' (C) clusters identified from largest to smallest population (CO-C15). Cluster identity and select conserved marker genes shown next to cluster colour key. c Comparison of organoid cell clustering in 'organoid only' to 'combined' clusters. Overlap in samples between clusters from the different analyses is shown using the Jaccard Index with a score of 1 (yellow) indicating identical clusters and 0 (blue) indicating no cells in common. $\mathbf{d}$ Number of cells contributing to each cluster from hFK and organoid samples. $\mathbf{e}$ Comparison of general cell type composition between organoid and hFK samples. Stroma includes C0, C1, C2, C3, and C9; nephron includes C6, C7, and C10. f Differentially expressed genes with largest fold changes between all organoid and all hFK cells. $\mathbf{g}$ Top conserved markers and differentially expressed genes between datasets for clusters from the 'combined' analysis. Cell cycle clusters not displayed. Similar analysis for nephron clusters is presented in Fig. 3

may have been subjected to suboptimal conditions prior to processing. We note this signature is only revealed after comparing to another dataset and is a potential drawback with using cells derived from primary tissue, which is otherwise an ideal reference. The ribosomal RNA signature enriched in kidney organoids may reflect a higher metabolic rate within kidney organoid cells in culture [45]. Most tissue culture media, including that used for kidney organoid culture, is high in glucose with culture conditions also physiologically hyperoxic compared to the fetus.

\section{Conserved and differential expression analysis between fetal and organoid cell types}

The major differences between the hFK and organoid datasets are likely to be technical artefacts due to sample 
isolation and culture conditions. To focus on biological differences between cell types, we removed the genes identified as sample-specific from subsequent differential expression analyses between the datasets. We then compared hFK and organoid cells within each cluster to assess conserved and differentially expressed genes (Additional files 4 and 6), summarised in Table 1 and Fig. $1 \mathrm{~g}$.

\section{Conserved stromal and endothelial cell types between organoid and fetal kidney}

Our understanding of stromal subpopulations in developing human kidneys is relatively poor. However, Lindstrom and colleagues sampled the cortex of the developing human kidney and classified the stromal cells within this sample into five populations representing three major groups: Lindstrom cluster (L) 9, marked by REN, MGP and GATA3; L2 marked by LUM, SFRP2 and DCN; and three grouped subpopulations L10-12 with markers including TCF21, ALDH1A2, ANGPT1, MEIS1, and TAGLN [10]. These populations appear to be conserved in kidney organoids with the combined cluster $\mathrm{C} 0$ correlating to the L10-12 clusters (conserved markers include TCF21, ALDH1A2, ANGPT1 and TAGLN); C1 representing L2 (conserved markers include LUM, SFRP2, and $D C N$ ); and C2 representing L9 (conserved markers include MGP and

Table 1 Summary of gene expression analyses for combined clusters excluding cell cycle and nephron lineage clusters

\begin{tabular}{|c|c|c|c|c|c|c|}
\hline \multicolumn{2}{|c|}{$\begin{array}{l}\text { Cluster and sample } \\
\text { origin }\end{array}$} & \multirow{3}{*}{$\begin{array}{l}\mathrm{DE} \\
3 \\
2\end{array}$} & \multirow{3}{*}{$\begin{array}{l}\text { Top enriched genes } \\
\text { CKS2, LBH, CCBE1 }\end{array}$} & \multirow[t]{3}{*}{$\begin{array}{l}\text { GO summary for } \geq 10 \\
\text { DE genes }\end{array}$} & \multirow{3}{*}{$\begin{array}{l}\text { Top cluster markers } \\
\text { SULT1E1, GPC3, MEG3, } \\
\text { SERPINH1, ALDH1A2, } \\
\text { TAGLN }\end{array}$} & \multirow{3}{*}{$\begin{array}{l}\text { Top conserved markers } \\
\text { TAGLN, ALDH1A2, PDGFRA, } \\
\text { ZEB2, ACTA2, ALX1, SNAI2, } \\
\text { COL6A3 }\end{array}$} \\
\hline Co Stroma & hFK & & & & & \\
\hline & Org & & & & & \\
\hline \multirow[t]{2}{*}{ C1 Stroma } & hFK & 87 & $\begin{array}{l}\text { SERPINI1, COL1A1, S100A10, } \\
\text { SFRP2, ANXA1, ASPN }\end{array}$ & $\begin{array}{l}\text { ECM organisation, } \\
\text { signalling receptor } \\
\text { binding }\end{array}$ & $\begin{array}{l}\text { DCN, COL1A1, LUM, IGF1, } \\
\text { POSTN, SFRP2, COL1A2, } \\
\text { OGN }\end{array}$ & $\begin{array}{l}\text { IGF1, SFRP2, COL1A1, DCN, } \\
\text { LUM, COL1A2, COL3A1, } \\
\text { POSTN }\end{array}$ \\
\hline & Org & 5 & RPL27A, PALLD, ACTA2, DKK1 & & & \\
\hline \multirow[t]{2}{*}{ C2 Stroma } & hFK & 11 & $\begin{array}{l}\text { REN, ID1, PDLIM1, } \\
\text { ITGA8, ENG, CPM }\end{array}$ & $\begin{array}{l}\text { Endothelial and smooth } \\
\text { muscle development }\end{array}$ & \multirow{2}{*}{$\begin{array}{l}\text { DLK1, MGP, NDUFA4L2, } \\
\text { GATA3, APOE, PDGFRB, } \\
\text { MEF2C }\end{array}$} & \multirow{2}{*}{$\begin{array}{l}\text { MGP, GATA3, NDUFA4L2, } \\
\text { MEF2C, ACTA2, PDGFRB, } \\
\text { PDLIM1 }\end{array}$} \\
\hline & Org & 2 & SSBP4, FTL & & & \\
\hline \multirow[t]{2}{*}{ C3 Stroma } & hFK & 23 & $\begin{array}{l}\text { ACTA2, NR2F1, ANGPT1, } \\
\text { ROBO2 }\end{array}$ & No significant results & \multirow{2}{*}{$\begin{array}{l}\text { MAB21L2, CXCL14, PRRX1, } \\
\text { ZFHX4, MAB21L1, CD24, } \\
\text { COL9A2 }\end{array}$} & \multirow{2}{*}{$\begin{array}{l}\text { ZFHX4, CTSK, HNRNPA1, } \\
\text { DNM3OS, SOX4, LIMA1, } \\
\text { TBX3 }\end{array}$} \\
\hline & Org & 12 & VIM, VAMP2, SNHG8, DKK1 & $\begin{array}{l}\text { Mitochondrial ribosome } \\
\text { binding }\end{array}$ & & \\
\hline \multirow[t]{2}{*}{ C4 Endothelium } & hFK & 45 & $\begin{array}{l}\text { FN1, RBP5, PLPP3, CCL21, } \\
\text { LGALS1, CXCL1, CXCL2, } \\
\text { CXCL8, ICAM1 }\end{array}$ & $\begin{array}{l}\text { Signalling receptor } \\
\text { binding, chemokine } \\
\text { activity }\end{array}$ & \multirow[t]{2}{*}{$\begin{array}{l}\text { GNG11, EGFL7, CLDN5, } \\
\text { ESAM, PVLAP, CD34, CAV1, } \\
\text { ARHGAP29, APLN }\end{array}$} & \multirow{2}{*}{$\begin{array}{l}\text { GNG11, EGFL7, CLDN5, } \\
\text { ESAM, PVLAP, S100A16, } \\
\text { ARHGAP29, APLNR, CAV1, } \\
\text { CD34, KDR, TIE1 }\end{array}$} \\
\hline & Org & 44 & $\begin{array}{l}\text { APLN, PECAM1, MMP1, } \\
\text { CAV1, CXCR4, HEY1 }\end{array}$ & $\begin{array}{l}\text { Angiogenesis, vascular } \\
\text { development }\end{array}$ & & \\
\hline \multirow[t]{2}{*}{ C9 Stroma } & hFK & 67 & $\begin{array}{l}\text { COL1A1, PLPP3, ITM2B, } \\
\text { IGF1, SPON2 }\end{array}$ & $\begin{array}{l}\text { Cell adhesion, ECM } \\
\text { organisation }\end{array}$ & \multirow[t]{2}{*}{$\begin{array}{l}\text { COL2A1, COL9A3, CNMD, } \\
\text { MIA, COL9A2, COL9A1, FIBIN }\end{array}$} & \multirow{2}{*}{$\begin{array}{l}\text { COL9A3, DCN, LUM, FIBIN, } \\
\text { COL1A2, OGN, IGFBP6, } \\
\text { COL1A1, SOX9, SFRP2, MGP }\end{array}$} \\
\hline & Org & 41 & $\begin{array}{l}\text { CNMD, PEG10, COL9A1, } \\
\text { COL9A3, GNG5, COL11A1 }\end{array}$ & $\begin{array}{l}\text { ECM organisation, } \\
\text { cartilage dev. }\end{array}$ & & \\
\hline \multirow[t]{2}{*}{ C11 Glia } & hFK & 203 & $\begin{array}{l}\text { S100B, PLP1, MPZ, } \\
\text { PMP22, ARHGAP15 }\end{array}$ & Axon development & \multirow[t]{2}{*}{$\begin{array}{l}\text { AP1S2, TTHY1, FABP7, } \\
\text { SOX2, MSX1, PCSK1N }\end{array}$} & \multirow{2}{*}{$\begin{array}{l}\text { S100B, GPM6B, TTYH1, PLP1, } \\
\text { SOX2, NKAIN3, PMP22, CNP, } \\
\text { VIM }\end{array}$} \\
\hline & Org & 136 & AP1S2, FABP7, ZFP36L1, MSX1 & $\begin{array}{l}\text { ATP synthesis, cell } \\
\text { respiration }\end{array}$ & & \\
\hline \multirow[t]{2}{*}{ C12 Immune } & hFK & 88 & $\begin{array}{l}\text { HLA-DPB1, HLA-DPA1, } \\
\text { HLA-DRB1, CXCR4, CD83 }\end{array}$ & $\begin{array}{l}\text { Response to IFN gamma, } \\
\text { antigen binding }\end{array}$ & \multirow{2}{*}{$\begin{array}{l}\text { HLA-DRA, CD74, SRGN, } \\
\text { S100A9, TYROBP, S100A8, } \\
\text { HLA-DPB1, LYZ }\end{array}$} & \multirow{2}{*}{$\begin{array}{l}\text { SRGN, LYZ, S100A9, TYROBP, } \\
\text { S100A8, FCER1G, SPP1, FTL, } \\
\text { CD74 }\end{array}$} \\
\hline & Org & 24 & PRTN3, MPO, CTSG, AZU1 & $\begin{array}{l}\text { Immune response, } \\
\text { defence response }\end{array}$ & & \\
\hline \multirow[t]{2}{*}{ C13 Blood } & hFK & 24 & $\begin{array}{l}\text { HBM, AHSP, ALAS2, } \\
\text { HEMGN, SLC25A37 }\end{array}$ & $\begin{array}{l}\text { Erythrocyte development, } \\
\text { oxygen transport }\end{array}$ & \multirow{2}{*}{$\begin{array}{l}\text { HBG1, HBB, HBA2, HBA1, } \\
\text { HMB, AHSP, ALAS2, SCNA, } \\
\text { GNG11, HEMGN }\end{array}$} & \multirow{2}{*}{$\begin{array}{l}\text { CYPC, SLC25A39, SLC25A37, } \\
\text { GPX1, HEY1, COPZ1, PRDX2, } \\
\text { ACTB, SELENBP1, PFN1 }\end{array}$} \\
\hline & Org & 168 & $\begin{array}{l}\text { FSCN1, EGFL7, FKBP1A, } \\
\text { EIF4G2, GNG5, TPM4, BAX }\end{array}$ & $\begin{array}{l}\text { Viral process, translation } \\
\text { initiation }\end{array}$ & & \\
\hline \multirow[t]{2}{*}{ C14 Neural } & hFK & N/A & & & \multirow{2}{*}{$\begin{array}{l}\text { HES6, CRABP1, TUBB2B, } \\
\text { STMN2, TAGLN3, SSTR2 }\end{array}$} & \multirow[t]{2}{*}{ N/A } \\
\hline & Org & N/A & & & & \\
\hline
\end{tabular}


GATA3). Thus, human kidney organoids contain stromal populations similar to hFK cortical stromal populations, which form part of the nephrogenic niche and have been shown to influence nephron formation in mice $[46,47]$. Few genes are differentially expressed between hFK and organoid cells in C0 but these include NRK and STMN2, upregulated in organoid cells, and CCBE1 and $L B H$ upregulated in hFK. More substantial differences are apparent in $\mathrm{C} 1$ with 87 genes upregulated in hFK compared to organoid cells including several signalling molecules such as SFRP2, IGF1, WNT2B, DKK2 and SEMA3A. The expression of $W N T 2 B$ is notable as this ligand is not expressed in the developing mouse kidney, and WNT signalling has several critical roles in kidney development. hFK cells within Cluster C3 express higher levels of markers associated with vascular smooth muscle and mesangial development including RENIN [48]. However, several highly upregulated markers are conserved within this cluster and the conserved signature also features several smooth muscle-associated genes such as GATA3, MEF2C, ACTA2, HOPX, ANGPT2 and PDGFRB. Stromal clusters C3 (marked by MAB21L2, CXCL14, PRRX1) and C9 (COL2A1, COL9A3, CNMD) had smaller contributions of hFK cells (less than $5 \%$ of total cells), which could indicate that these organoid stromal clusters are less similar to native cell types, or that equivalent stromal populations are not adequately represented in the hFK sample. In situ hybridisation results from the mouse embryo indicate that some of the most upregulated markers of organoid stromal cell types $\mathrm{C} 3$ and $\mathrm{C} 9$ are expressed in the medullary and ureteric stroma of the developing kidney (Additional file 1), which was not sampled in the hFK data.

The endothelial cluster $\mathrm{C} 4$ featured extensive conservation of established markers such as CLDN5, CDH5, CD34, KDR, TIE1, SOX17, SOX7 and FLT1. GO analysis of conserved markers resulted in terms related to vascular development indicating congruence between organoid endothelial cells and those from the hFK data. Despite this conservation, several genes were enriched in hFK or organoid endothelial cells. hFK upregulated genes were associated with cell signalling including chemokines CXCL1, CXCL2 and CXCL8 whereas organoid cells expressed higher levels of endothelial markers such as PLXND1, APLN and PECAM1. As such organoid endothelial cells appear to be appropriately specified but differ in the complement of signalling molecules they express. Whether this is the result of cell intrinsic factors or a response to being embedded in a different stromal environment is unclear.

\section{Glial, neural, immune and blood clusters}

Some clusters in the combined analysis consisted mainly of cells from one dataset. Organoid-enriched clusters include a glial cluster $\mathrm{C} 11$ (marked by TTYH1, FABP7,
SOX2), which included less than ten hFK cells, and C14 (marked by SOX11, ELAVL3, ELAVL4) that mostly contained organoid cells. GO analysis of the top markers of these clusters identifies terms associated with glial cell differentiation (C11) and generation of neurons (C14). There is some evidence of neural precursors being present during mouse [49] and human [43] kidney development, and neural populations play an important role in adult renal physiology. However, neural and renal progenitors have distinct embryonic origins, the former from the ectoderm and the latter from the intermediate mesoderm $[6,50,51]$. As our organoid protocol directs the bulk of cells towards an intermediate mesoderm-like fate [3], these glial and neural cell types are considered off-target and may reflect cells that adopted an alternative identity during the early stages of differentiation and persisted in culture.

An immune cell cluster (C12, marked by PTPRC, TYR$O B P, F C E R 1 G)$ was mostly derived from hFK cells but surprisingly, 20 organoid cells contributed to this cluster and shared expression of immune response genes. During development, haematopoietic progenitors, cells can arise from specialised endothelial cells termed hemogenic endothelium [52]. This type of endothelium occurs within the aorta-gonad-mesonephros region [53], which is adjacent to the site of kidney development. Markers of hemogenic endothelial cells such as PECAM1, KDR, KIT and $C D H 5$ [52] are expressed in the organoid vasculature and, as such, it is possible that kidney organoids have some capacity to generate cells involved in the immune response.

Top markers of cluster $\mathrm{C} 13$ included genes highly expressed in blood (HBG1, $H B B, H B A 2)$, a signature primarily driven by the hFK-derived cells within the cluster as organoid-derived cells within this cluster did not share expression of these cell type-specific markers. GO analysis of upregulated organoid genes, and genes that were conserved markers between fetal and organoid cells in this cluster led to terms that were not related to blood.

\section{Conserved nephron progenitor and early nephron cell types} hFK and organoid cells were present in clusters representing nephron progenitor cells (C6) and nephron epithelium (C10), but other hFK cells were split between a cluster containing most organoid podocytes (C7) and a hFK-specific podocyte cluster (C15).

\section{Congruence and differences between hFK and kidney organoid nephron cell types}

To compare nephron subpopulations in detail, we reclustered cells from the combined nephron lineage clusters $(\mathrm{C} 6, \mathrm{C} 7, \mathrm{C} 10, \mathrm{C} 15)$ in isolation. This generated seven combined nephron $(\mathrm{CN})$ clusters with cells from hFK and organoid co-clustering within populations representing nephron progenitors $(\mathrm{CN} 2)$, differentiating 
nephron progenitors $(\mathrm{CN} 1)$, distal $(\mathrm{CN} 4)$ and proximal (CN5) nephron segments, podocyte precursor (CN3) and podocyte cells (CNO). The hFK-specific podocyte cluster (CN7) noted previously was maintained in this analysis, and a new cluster of hFK stromal cells, marked by COL3A1, POSTN and MEG3 (CN6) was resolved. CN6 does not express any known nephron markers aside from
TMEM100, which was reported to be specific to nephron progenitors in the human fetal kidney [10]. In the absence of other nephron progenitor markers, this cluster does not appear to be part of the nephron lineage (Fig. 3a, b). Cells within this stromal $\mathrm{CN} 6$ cluster may have been associated with the nephron lineage based on the broad expression of stromal markers within human nephron progenitor
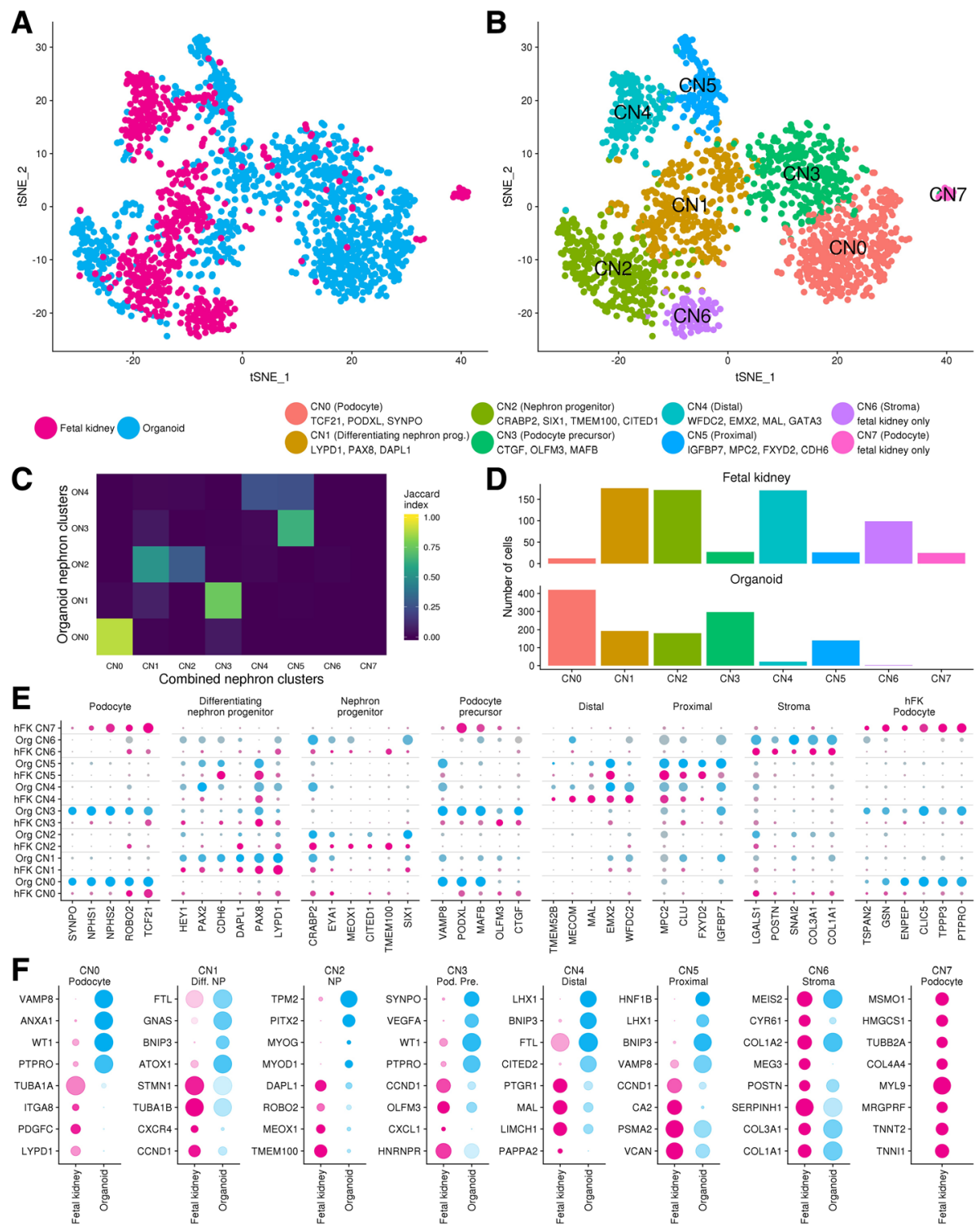

Fig. 3 Comparison of nephron cell types within kidney organoids and human fetal kidney. $\mathbf{a}$, $\mathbf{b}$ Sample of origin and re-clustering of combined nephron (CN) lineage cells results in eight clusters. Cluster identity and select conserved marker genes shown next to cluster colour key. Cells for this analysis were selected from combined clusters C6, C7, C10 and C15. c Comparison of organoid cells between organoid nephron (ON) and combined nephron (CN) clusters. Colours show overlap in cells between clusters according to the Jaccard Index. $\mathbf{d}$ Number of cells in each combined nephron cluster by dataset. e Split dot plot showing relative expression for select marker genes within organoid and hFK cells in the combined nephron clusters. hFK data in pink, organoid in blue. Circle size represents the proportion of cells in the cluster expressing that gene, shading indicates expression level (low to high reflected as light to dark). f Top differentially expressed genes between datasets within combined nephron clusters. Chart colouring and shading as per e. Results for CN6 and CN7 are not differential expression results as few (CN6) or no (CN7) organoid cells are present within these clusters. These instead reflect top cluster markers (CN6) or markers enriched in CN7 but not CN0 or CN3. Organoid expression values for CN6 are derived from three organoid cells within this cluster 
cells [10]. Again, organoid nephron cells formed similar groups when clustered with the hFK data as when clustered alone (Fig. 3c). hFK and organoid cells contributed to most clusters with the largest contributions in the nephron progenitor and differentiating progenitor cluster (Fig. 3d). hFK cells were scattered through the two organoid podocyte clusters but again a group of hFK podocyte cells remained separate. In the clusters representing the nephron epithelium, organoid cells were located in the proximal nephron cluster with few cells contributing to the distal while the hFK cells displayed the opposite pattern, consistent with the previous hFK analysis [10] and the under representation of distal nephron in these organoid samples.

Key cell-type markers were conserved between the datasets but differential expression testing revealed underlying differences in transcriptional profiles between hFK and organoid nephron cell types (Table 2, Fig. 3e, f, Additional files 7 and 8). hFK cells within nephron progenitor clusters showed enhanced expression of progenitor genes TMEM100, MEOX1, ROBO2 and $D A P L 1$, and a small portion of organoid nephron progenitors expressed low levels of muscle progenitor genes MYOD1 and MYOG (Additional file 1). Thus, despite the conservation of key markers, this population may be adopting a muscle progenitor fate in the absence of appropriate signals to reinforce nephron progenitor identity. Alternatively, a small number of muscle progenitors may have clustered with the nephron progenitors due to similarities in their expression profiles. Likewise, underlying a conserved profile, hFK cells within the differentiating nephron progenitor cluster expressed elevated levels of proliferation associated genes such as CCND1 and PCLAF likely reflecting higher proliferation rates as seen during commitment to nephron formation in vivo [54].

Most of the top markers of the nephron tubule clusters represented established markers of distal and proximal tubule and were conserved between hFK and organoid cells. hFK cells expressed higher levels of distal markers MAL, LIMCH1 and PAPPA2 in distal cluster $\mathrm{CN} 4$ and organoid cells in proximal cluster CN5 had increased expression of genes associated with ureteric bud and pronephros development.

Table 2 Summary of gene expression analyses for combined nephron clusters excluding stromal cluster CN6

\begin{tabular}{|c|c|c|c|c|c|c|}
\hline $\begin{array}{l}\text { Cluster and } \\
\text { sample origin }\end{array}$ & & DE & Top enriched genes & $\begin{array}{l}\text { GO summary for } \geq 10 \\
\text { DE genes }\end{array}$ & Top cluster markers & Top conserved markers \\
\hline \multirow[t]{2}{*}{ CNO Pod } & hFK & 79 & $\begin{array}{l}\text { TUB1A1, NR2F1, SOX4, } \\
\text { LGALS1, NUSAP1, LYPD1 }\end{array}$ & $\begin{array}{l}\text { Central nervous system } \\
\text { development }\end{array}$ & \multirow{2}{*}{$\begin{array}{l}\text { S100A6, TCF21, PODXL, } \\
\text { TPPP3, SBSPON, MMP5, } \\
\text { CPXM1, DUSP23, THSD7A, } \\
\text { MME }\end{array}$} & \multirow{2}{*}{$\begin{array}{l}\text { TCF21, MME, PODXL, } \\
\text { THSD7A, TPPP3, SBSPON, } \\
\text { MAFB, ENPEP, ROBO2, } \\
\text { NPHS2 }\end{array}$} \\
\hline & Org & 175 & $\begin{array}{l}\text { VAMP8, ANXA1, AIF1, } \\
\text { S100A4, TGFBR3, WT1 }\end{array}$ & $\begin{array}{l}\text { ATP synthesis, oxidative } \\
\text { phosphorylation }\end{array}$ & & \\
\hline \multirow[t]{2}{*}{ CN1 Diff. NP } & hFK & 37 & $\begin{array}{l}\text { CCND1, PCNA, CXCR4, } \\
\text { TUBA1A, TBUA1B, STMN1 }\end{array}$ & mRNA splicing & \multirow{2}{*}{$\begin{array}{l}\text { LYPD1, PAX8, HIST1H4C, } \\
\text { PCLAF, CDH6, DAPL1, } \\
\text { CCND1, RBP1, HMGB2 }\end{array}$} & \multirow{2}{*}{$\begin{array}{l}\text { LYPD1, PAX8, HIST1H4C, } \\
\text { PCLAF, DAPL1, PCP4, } \\
\text { HEY1, CDH6, RBP1, PAX2 }\end{array}$} \\
\hline & Org & 4 & FTL, GNAS, BNIP, ATOX1 & & & \\
\hline \multirow[t]{2}{*}{ CN2 NP } & hFK & 24 & $\begin{array}{l}\text { TMEM100, ITM2C, MEOX1, } \\
\text { EPCAM, ROBO2, DAPL1 }\end{array}$ & $\begin{array}{l}\text { Amyloid precursor } \\
\text { biosynthesis }\end{array}$ & \multirow{2}{*}{$\begin{array}{l}\text { ACTC1, NNAT, MYLPF, } \\
\text { MYL1, TMEM100, TPM2, } \\
\text { CRABP2, TUB1A1 }\end{array}$} & \multirow{2}{*}{$\begin{array}{l}\text { NNAT, CRABP2, TUB1A1, } \\
\text { IGF2, SIX1, TMEM100, } \\
\text { CITED1, SOX4, MEOX1, } \\
\text { MEIS2 }\end{array}$} \\
\hline & Org & 25 & $\begin{array}{l}\text { ACT1C1, MYLPF, TPM2, } \\
\text { PITX2, MYOG, MYOD }\end{array}$ & Muscle filament sliding & & \\
\hline \multirow[t]{2}{*}{ CN3 Pod. Pre. } & hFK & 37 & $\begin{array}{l}\text { CCND1, OLFM3, CXCL1, } \\
\text { HNRNPR, STMN1 }\end{array}$ & No significant BP terms & \multirow{2}{*}{$\begin{array}{l}\text { CTGF, GPX3, TSPAN8, } \\
\text { PAPPA, PAPPA, ITIH5, } \\
\text { SERINC5, HES4, NPHS2, } \\
\text { NPHS1, MAFB, PTPRO }\end{array}$} & \multirow{2}{*}{$\begin{array}{l}\text { CTGF, OLFM3, BCAM, } \\
\text { NPHS1, ARHGAP29, CLDN1, } \\
\text { LEPROT, TMP1, STON2, } \\
\text { CLDN5, MAFB }\end{array}$} \\
\hline & Org & 78 & $\begin{array}{l}\text { GPX3, ANXA1, S100A4, AIF1, } \\
\text { PTPRO, SYNPO, VEGFA, WT1 }\end{array}$ & $\begin{array}{l}\text { Epithelial cell diff. involved } \\
\text { in kidney development }\end{array}$ & & \\
\hline \multirow[t]{2}{*}{ CN4 Distal } & hFK & 26 & $\begin{array}{l}\text { PTGR1, MAL, LIMCH1, ELF3, } \\
\text { ALDH1A1, PAPPA2 }\end{array}$ & Epithelium development & \multirow{2}{*}{$\begin{array}{l}\text { HBG2, ATF3, LIMCH1, } \\
\text { MAL, WFDC2, BTG2, } \\
\text { HES1, KLF6, MECOM, } \\
\text { ELF3, TUBB2B, GATA3 }\end{array}$} & \multirow{2}{*}{$\begin{array}{l}\text { WFDC2, EMX2, LIMCH1, } \\
\text { MAL, TUBB2B, SAT1, } \\
\text { MECOM, HMGA1, HES1, } \\
\text { GATA3, ATP1B1, GNG11 }\end{array}$} \\
\hline & Org & 11 & $\begin{array}{l}\text { LHX1, BNIP3, FTL, CKB, BASP1, } \\
\text { CITED2, HNRNPAB }\end{array}$ & Kidney morphogenesis & & \\
\hline \multirow[t]{2}{*}{ CN5 Proximal } & hFK & 34 & $\begin{array}{l}\text { CCND1, CA2, VCAN, ELF3, } \\
\text { DCDC2, FLRT3 }\end{array}$ & No significant BP terms & \multirow{2}{*}{$\begin{array}{l}\text { IGFBP7, CD24, PCP4, } \\
\text { PCSK1N, FXYD2, EMX2, } \\
\text { MPC2, APOE, CLU, } \\
\text { CFAP126, FTL, ATP1B1 }\end{array}$} & \multirow{2}{*}{$\begin{array}{l}\text { IGFBP7, MPC2, SMIM24, } \\
\text { FLRT3, EMX2, FXYD2, } \\
\text { GNG11, TSPAN12, CLU, } \\
\text { PCP4, ATP1B1, PDZK1 }\end{array}$} \\
\hline & Org & 14 & $\begin{array}{l}\text { PCSK1N, CITED2, BNIP3, } \\
\text { S100A13, MLLT1, PRDX5, } \\
\text { VAMP8, LHX1 }\end{array}$ & $\begin{array}{l}\text { Ureteric bud morphogenesis, } \\
\text { pronephros development }\end{array}$ & & \\
\hline \multirow[t]{2}{*}{ CN7 hFK Pod } & hFK & & & & \multirow{2}{*}{$\begin{array}{l}\text { CXCL12, TNNI1, TNNT2, } \\
\text { MME, MYL9, MRGPRF, } \\
\text { TPPP3, ANXA2, COL4A4, } \\
\text { ADM, PTPRO, MSMO1 }\end{array}$} & \multirow[t]{2}{*}{ N/A } \\
\hline & Org & & & & & \\
\hline
\end{tabular}

Abbreviations: $D E$ differentially expressed (adjusted $p$ value $<0.05$, absolute log fold change greater than 0.8 ), $h F K$ human fetal kidney, Org organoid. GO summary reporting top significant Gene Ontology (GO) Biological process (BP) results when fetal kidney or organoid DE gene lists were greater than or equal to ten genes. Full lists of cluster markers, conserved and differentially expressed genes and corresponding GO analyses available in Additional files or on website [32] 
Of the three podocyte clusters, CN3 featured conserved expression of recently defined human podocyte precursor markers MAFB, CTGF and OLFM3 [38] though OLFM3 was expressed at higher levels in hFK cells in this cluster and organoid cells had higher levels of several podocyte markers such as PTPRO, SYNPO, VEGFA and WT1. hFK and organoid cells in $\mathrm{CNO}$ expressed podocyte markers TCF21, POXDL, ROBO2 and NPHS2 but hFK cells within this cluster maintained expression of human nephron progenitor markers LGALS1 and LYPD1 [38] suggesting these cells may represent a more progenitor-like state than the organoid cells in this cluster. A final hFK-specific podocyte cluster formed distinct from the other two clusters which still included podocyte markers such as PTPRO and TCF 21 as marker genes but also included genes such as TNNI1, TNNT2 and MYL9, which are expressed in cardiac muscle and podocytes. As markers of $\mathrm{CNO}$ and $\mathrm{CN} 7$ largely overlap and represent a maturing podocyte state (POXDL, PTPRO, TCF21 AIF1), we investigated disparities between these clusters by performing differential expression analysis (Additional file 9).

Podocyte genes COL4A4 and ANXA2 as well as TNNI1, TUBA1A, COL9A1, STMN1 and CA2 were upregulated in hFK-specific CN7. Genes upregulated in CNO included podocyte enriched genes ANXA1, GPX3, VAMP8, DACH1 and WT1 as well as additional genes related to ATP synthesis that likely relate to the increased growth rate in culture. While CN0 expresses established podocyte markers, this cluster may also contain podocyte precursor states or podocytes that have not yet matured into glomeruli. Such a distinction may underlie the separation between CNO and CN7.

This comparative analysis of nephron cell types within kidney organoids and human fetal kidney shows strong conservation of key cell type-specific markers while uncovering differences in the expression levels of key nephron progenitor markers, and a separation of some hFK podocytes from others, potentially reflecting in vivo maturation. We did not observe differences in transporter expression or markers of tubule maturation between organoid and hFK samples but that may be due to insufficient depth of profiling in these scRNA-seq datasets.

\section{Discussion}

We performed an in-depth analysis of nephron subpopulations in kidney organoids and found co-expression of a robust suite of established cell type-specific markers. Pseudotime analysis of these cell types suggests organoid nephron formation replicates an expected developmental trajectory from nephron progenitor to podocyte and tubular end points. We then asked whether there were underlying differences between kidney organoid cell types and equivalent populations in the developing human kidney. Organoid and hFK single-cell RNA-seq datasets were integrated and clustered, with cells from both datasets contributing to most clusters. Conserved gene expression between organoid and hFK cells within endothelial, stromal and nephron cell types revealed congruence between these cell types demonstrating the capacity of organoids to represent many aspects of the developing human kidney. Where immunofluorescence had identified the presence of stromal markers in organoids, our single-cell analysis identified five stromal subpopulations, at least three of which are conserved to some level in the developing human kidney. The remaining two may represent renal stromal populations that are simply not represented in the hFK data set due to limited tissue collection, or off-target stromal cell types. Further comparisons with more complete human data sets will be required to discern between these options.

A recent study from $\mathrm{Wu}$ et al. also used single-cell analysis to analyse our kidney organoid protocol across time and compare it to another organoid protocol and human kidney cell types [55]. While the renal populations and congruence with human kidney cell types we identify are consistent with those results, the relative proportion and types of cells captured by that analysis differs somewhat from our findings. For example, we detect a muscle progenitor population, where they detected a cluster of melanocytes. We detect a glial and a neural cluster and they detected a neural progenitor population and four neuron clusters. The proportion of off-target populations in those samples is higher than in our analysis ( $20 \% \mathrm{Wu}$ et al., $6 \%$ this study), as is the proportion of tubule to podocyte cells. As such, the proportions of cell types generated by the same differentiation protocol are likely to vary between laboratories.

We were unable to resolve a distinct population representing ureteric epithelium in either dataset; however, markers previously used to define this population were under represented in the organoids analysed in this study. We previously reported simultaneous generation of presumptive ureteric epithelium and nephron lineages, with the proportions of cell types generated dependent on the timing of exposure to signals that pattern the anterior-posterior axis of the intermediate mesoderm [3]. The organoids generated for the present study were distinctly posterior and hence contained a lower frequency of epithelial GATA3 $^{+}$structures. Recent studies argue that ureteric epithelium and nephron lineages cannot be generated simultaneously because they arise from distinct regions during embryonic development and instead must be generated using distinct protocols [7]. Analysis of this epithelial GATA $3^{+}$cluster from other kidney organoids is required to further explore the identity of this cell type. However, the absence of any ureteric epithelial cluster in the hFK data suggests a resistance of this tubular epithelium to dissociate into single cells. This same population 
may be resistant to single-cell isolation from organoids. Hence, this will need to be overcome, perhaps using alternative technologies such as nuclear RNA preparations.

Differential expression within combined organoid and fetal kidney clusters identified an upregulation of ribosomal genes in kidney organoids and a heat shock/unfolded protein response in the fetal kidney data, both likely the result of technical artefacts rather than fundamental differences in cell identity. Examining gene expression after excluding these sample-enriched genes revealed additional differences between hFK and organoid cell types including notable changes in the levels of expression of nephron progenitor marker genes and in the levels and repertoire of growth factors expressed by stromal and endothelial cells. What was not evident in either dataset was the expression of several cell type-specific ligands and receptors that are apparent in analogous single cell datasets from the developing mouse kidney $[33,56]$. For example, genes such as GDNF and RET, which encode a key ligand and receptor pair are known to operate in human kidney development as both genes cause renal birth defects when mutated in humans [57]. Being unable to detect the expression of such important genes in the reference hFK dataset leaves the possibility of important differences between organoid and hFK cell types that may only be revealed with deeper profiling.

\section{Conclusions}

This analysis supports a conservation of cell types between organoids and human fetal kidney. Overall, the data presented here builds confidence in the fidelity of organoid nephron, stromal and endothelial cell types, which will encourage disease modelling and drug screening efforts in human kidney organoids.

\section{Additional files}

Additional file 1: Supporting analysis for organoid data set. Figure with quality control, integration and supporting analysis for organoid dataset. (PNG $7123 \mathrm{~kb}$ )

Additional file 2: Organoid cluster markers. Organoid cluster markers and associated GO terms. (XLSX $3062 \mathrm{~kb}$ )

Additional file 3: Organoid nephron cluster markers. Organoid nephron cluster markers and associated GO terms. (XLSX $1089 \mathrm{~kb})$

Additional file 4: Combined conserved markers. Markers conserved between organoid and hFK cells within combined clusters and associated GO terms. (XLSX $77 \mathrm{~kb})$

Additional file 5: Differential expression analysis between organoid and hFK samples. Results from differential expression analysis between organoid and hFK samples used to identify a signature representing the main differences between the organoid and hFK datasets. (XLSX $2050 \mathrm{~kb}$ )

Additional file 6: Differential expression analysis for combined clusters. Results from differential expression analysis for organoid and hFK cells within combined clusters. (XLSX $2893 \mathrm{~kb}$ )

Additional file 7: Combined nephron conserved markers. Combined nephron cluster markers and associated GO terms. (XLSX $673 \mathrm{~kb}$ )
Additional file 8: Differential expression analysis for combined nephron clusters. Results from differential expression testing between organoid and hFK cells within each combined nephron cluster after removal of the sample-enriched signature. (XLSX $2040 \mathrm{~kb}$ )

Additional file 9: Differential expression analysis between podocytes in CNO vs CN7. Results for differential gene expression testing between hFKspecific podocyte cluster CN7 and mixed organoid and hFK podocyte cluster CNO. (XLSX 77 kb)

\section{Acknowledgements}

Single cell sequencing was performed at the Australian Genome Research Facility Genomics Innovation Hub with the assistance of J. Jabbari and A. Seidi. Microscopy was performed at the Murdoch Children's Research Institute. We would like to thank D. Newgreen, D. Zhang, K.N. North, P.J. Houweling, S.M Wilson, J.M. Vanslambrouck, S.E. Howden, and K.S. Tan from the Murdoch Children's Research Institute for assisting in generation of organoids or providing antibody reagents.

\section{Funding}

This work was supported by the Australian Research Council (DE150100652), the National Health and Medical Research Council (NHMRC) of Australia (GNT1156567), National Institutes of Health Rebuilding a Kidney consortium (DK107344) and seed funding from the Murdoch Children's Research Institute and the University of Melbourne. A.N.C. was supported by a Discovery Early Career Researcher Award from the Australian Research Council. M.H.L. is a Senior Principal Research Fellow of the NHMRC (GNT1136085). A.O. is a Career Development Fellow of the NHMRC. L.Z. is supported by an Australian Government Research Training Program (RTP) Scholarship. MCRI is supported by the Victorian Government's Operational Infrastructure Support Program. These funding bodies had no role in any aspect of the study design, execution, or writing.

\section{Availability of data and materials}

Both organoid datasets are available from GEO accession number GSE114802 [58] and the Lindstrom fetal kidney dataset is available from GEO accession GSE102596 [59]. A website showing reports produced during analysis, including the exact software versions and parameters used, can be accessed at http://oshlacklab.com/combes-organoid-paper/ and the analysis code is available at https://github.com/Oshlack/combes-organoid-paper [32].

\section{Authors' contributions}

PE performed the organoid differentiations, single-cell isolation and immunofluorescence. LZ designed and performed the single-cell analysis and generated figures under the supervision of AO. ANC designed and performed the single-cell experiments and immunofluorescence and was primarily responsible for data interpretation and writing, under the supervision of MHL. All authors contributed intellectually to the project and manuscript revisions. All authors read and approved the final manuscript.

\section{Ethics approval and consent to participate}

This study includes published, publically available data from consented, anonymized, human fetal tissue, obtained from elective terminations following review of the study by Keck School of Medicine of the University of Southern California's Institutional Review Board and in accordance with the Declaration of Helsinki [10].

\section{Consent for publication}

Not applicable.

\section{Competing interests}

M.H.L. has consulted for and received funding from Organovo Holdings. The remaining authors declare that they have no competing interests.

\section{Publisher's Note}

Springer Nature remains neutral with regard to jurisdictional claims in published maps and institutional affiliations. 


\section{Author details}

'Department of Anatomy \& Neuroscience, University of Melbourne, Melbourne, VIC, Australia. 'Murdoch Children's Research Institute, Melbourne, VIC, Australia. ${ }^{3}$ School of Biosciences, The University of Melbourne, Melbourne, VIC, Australia. ${ }^{4}$ Department of Paediatrics, The University of Melbourne, Melbourne, VIC, Australia.

Received: 24 September 2018 Accepted: 14 January 2019

Published online: 23 January 2019

\section{References}

1. McCauley HA, Wells JM. Pluripotent stem cell-derived organoids: using principles of developmental biology to grow human tissues in a dish. Development. 2017;144(6):958-62.

2. Takasato M, Er PX, Becroft M, Vanslambrouck JM, Stanley EG, Elefanty AG, et al. Directing human embryonic stem cell differentiation towards a renal lineage generates a self-organizing kidney. Nat Cell Biol. 2014;16(1):118-26.

3. Takasato M, Er PX, Chiu HS, Maier B, Baillie GJ, Ferguson C, et al. Kidney organoids from human iPS cells contain multiple lineages and model human nephrogenesis. Nature. 2015;526(7574):564-8.

4. Freedman BS, Brooks CR, Lam AQ, Fu H, Morizane R, Agrawal V, et al. Modelling kidney disease with CRISPR-mutant kidney organoids derived from human pluripotent epiblast spheroids. Nat Commun. 2015;6:8715.

5. Morizane R, Lam AQ, Freedman BS, Kishi S, Valerius MT, Bonventre JV. Nephron organoids derived from human pluripotent stem cells model kidney development and injury. Nat Biotechnol. 2015:33(11):1193-200.

6. Taguchi A, Kaku Y, Ohmori T, Sharmin S, Ogawa M, Sasaki H, et al. Redefining the in vivo origin of metanephric nephron progenitors enables generation of complex kidney structures from pluripotent stem cells. Cell Stem Cell. 2014;14(1):53-67.

7. Taguchi A, Nishinakamura R. Higher-order kidney organogenesis from pluripotent stem cells. Cell Stem Cell. 2017;21(6):730-46 e6.

8. Przepiorski A, Sander V, Tran T, Hollywood JA, Sorrenson B, Shih JH, et al. A simple bioreactor-based method to generate kidney organoids from pluripotent stem cells. Stem Cell Reports. 2018;11(2):470-84.

9. Phipson B, Er PX, Combes AN, Forbes TA, Howden SE, Zappia L, et al. Evaluation of variability in human kidney organoids. Nat Methods. 2019; 16(1):79-87.

10. Lindstrom NO, Guo J, Kim AD, Tran T, Guo Q, De Sena BG, et al. Conserved and divergent features of mesenchymal progenitor cell types within the cortical nephrogenic niche of the human and mouse kidney. J Am Soc Nephrol. 2018;29(3):806-24

11. Takasato M, Er PX, Chiu HS, Little MH. Generation of kidney organoids from human pluripotent stem cells. Nat Protoc. 2016;11(9):1681-92.

12. Briggs JA, Sun J, Shepherd J, Ovchinnikov DA, Chung TL, Nayler SP, et al. Integration-free induced pluripotent stem cells model genetic and neural developmental features of Down syndrome etiology. Stem Cells. 2013;31(3):467-78

13. R Core Team. R: A language and environment for statistical computing: $R$ Foundation for Statistical Computing, Vienna, Austria.; 2013. Available from: URL http://www.R-project.org/.

14. Lun A, Risso D. SingleCellExperiment: S4 Classes for Single Cell Data. bioconductor.org2017.

15. Smedley D, Haider S, Durinck S, Pandini L, Provero P, Allen J, et al. The BioMart community portal: an innovative alternative to large, centralized data repositories. Nucleic Acids Res. 2015;43(W1):W589-W98.

16. Durinck S, Moreau Y, Kasprzyk A, Davis S, De Moor B, Brazma A, et al. BioMart and Bioconductor: a powerful link between biological databases and microarray data analysis. Bioinformatics. 2005:21(16):3439-40.

17. Scialdone A, Natarajan KN, Saraiva LR, Proserpio V, Teichmann SA, Stegle O, et al. Computational assignment of cell-cycle stage from single-cell transcriptome data. Methods. 2015;85:54-61.

18. Lun AT, McCarthy DJ, Marioni JC. A step-by-step workflow for low-level analysis of single-cell RNA-seq data with Bioconductor. F1000Research. 2016;5:2122.

19. McCarthy DJ, Campbell KR, Lun AT, Wills QF. Scater: pre-processing, quality control, normalization and visualization of single-cell RNA-seq data in R. Bioinformatics. 2017:33(8):1179-86.

20. Butler A, Hoffman P, Smibert P, Papalexi E, Satija R. Integrating single-cell transcriptomic data across different conditions, technologies, and species. Nat Biotechnol. 2018;36(5):411-20.
21. Satija R, Farrell JA, Gennert D, Schier AF, Regev A. Spatial reconstruction of single-cell gene expression data. Nat Biotechnol. 2015;33(5):495-502.

22. Hardoon DR, Szedmak S, Shawe-Taylor J. Canonical correlation analysis: an overview with application to learning methods. Neural Comput. 2004;16(12): 2639-64.

23. Hotelling H. Relations between two sets of variates. Biometrika. 1936;28: 321-77.

24. Berndt DJ, Clifford J. Using dynamic time warping to find patterns in time series. Proceedings of the 3rd International Conference on Knowledge Discovery and Data Mining; 1994.

25. Blondel VD, Guillaume JL, Lambiotte R, Lefebvre E. Fast unfolding of communities in large networks. J Stat Mech-Theory E. 2008;2008:10008 http://iopscience.iop.org/article/10.1088/1742-5468/2008/10/P10008/meta.

26. Zappia L, Oshlack A. Clustering trees: a visualization for evaluating clusterings at multiple resolutions. GigaScience. 2018;7(7):giy083.

27. Bauer DF. Constructing confidence sets using rank statistics. J Am Stat Assoc. 1972;67(339):687-90.

28. Qiu X, Mao Q, Tang Y, Wang L, Chawla R, Pliner HA, et al. Reversed graph embedding resolves complex single-cell trajectories. Nat Methods. 2017; 14(10):979-82.

29. Trapnell C, Cacchiarelli D, Grimsby J, Pokharel P, Li S, Morse M, et al. The dynamics and regulators of cell fate decisions are revealed by pseudotemporal ordering of single cells. Nat Biotechnol. 2014;32(4):381-6.

30. Wickham H. ggplot2: Elegant Graphics for Data Analysis. 2 ed. New York: Springer International Publishing; 2016. p. 260.

31. Wilke CO. cowplot: Streamlined Plot Theme and Plot Annotations for 'ggplot2.' 2018 [May 21, 2018]. Available from: https://CRAN.R-project.org/ package $=$ cowplot

32. Zappia L, Combes AN, Er PX, Little M, Oshlack A. Combes organoid paper analysis code. GitHub. 2018. Available from: http://oshlacklab.com/combesorganoid-paper/. Accessed 12 Jan 2018.

33. Combes AN, Phipson B, Zappia L, Lawlor KT, Er PX, Oshlack A, et al. High throughput single cell RNA-seq of developing mouse kidney and human kidney organoids reveals a roadmap for recreating the kidney. bioRxiv. 2017; 332:273-86

34. Georgas K, Rumballe B, Valerius MT, Chiu HS, Thiagarajan RD, Lesieur E, et al. Analysis of early nephron patterning reveals a role for distal RV proliferation in fusion to the ureteric tip via a cap mesenchyme-derived connecting segment. Dev Biol. 2009;332(2):273-86.

35. Thiagarajan RD, Georgas KM, Rumballe BA, Lesieur E, Chiu HS, Taylor D, et al. Identification of anchor genes during kidney development defines ontological relationships, molecular subcompartments and regulatory pathways. PLoS One. 2011;6(2):e17286.

36. Chen J, Bardes EE, Aronow BJ, Jegga AG. ToppGene Suite for gene list enrichment analysis and candidate gene prioritization. Nucleic Acids Res. 2009;37(Web Server issue):W305-11.

37. Brunskill EW, Potter SS. Changes in the gene expression programs of renal mesangial cells during diabetic nephropathy. BMC Nephrol. 2012;13:70.

38. Lindstrom NO, De Sena BG, Tran T, Ransick A, Suh G, Guo J, et al. Progressive recruitment of mesenchymal progenitors reveals a timedependent process of cell fate acquisition in mouse and human nephrogenesis. Dev Cell. 2018;45(5):651-60 e4.

39. O'Brien LL, Guo Q, Lee Y, Tran T, Benazet JD, Whitney PH, et al. Differential regulation of mouse and human nephron progenitors by the six family of transcriptional regulators. Development. 2016;143(4):595-608.

40. Phipson B, Er PX, Combes AN, Forbes TA, Howden SE, Zappia L, et al. Transcriptional evaluation of the developmental accuracy, reproducibility and robustness of kidney organoids derived from human pluripotent stem cells. Nature Methods. 2018.

41. Takasato M, Er PX, Chiu HS, Maier B, Baillie GJ, Ferguson C, et al. Kidney organoids from human iPS cells contain multiple lineages and model human nephrogenesis. Nature. 2016;536(7615):238.

42. Kobayashi A, Mugford JW, Krautzberger AM, Naiman N, Liao J, McMahon AP. Identification of a multipotent self-renewing stromal progenitor population during mammalian kidney organogenesis. Stem Cell Reports. 2014;3(4):650-62.

43. Young MD, Mitchell TJ, Vieira Braga FA, Tran MGB, Stewart BJ, Ferdinand JR, et al. Single-cell transcriptomes from human kidneys reveal the cellular identity of renal tumors. Science. 2018;361(6402):594-9.

44. Adam M, Potter AS, Potter SS. Psychrophilic proteases dramatically reduce single-cell RNA-seq artifacts: a molecular atlas of kidney development. Development. 2017;144(19):3625-32. 
45. Rudra D, Warner JR. What better measure than ribosome synthesis? Genes Dev. 2004;18(20):2431-6.

46. Das A, Tanigawa S, Karner CM, Xin M, Lum L, Chen C, et al. Stromalepithelial crosstalk regulates kidney progenitor cell differentiation. Nat Cell Biol. 2013;15(9):1035-44.

47. Fetting JL, Guay JA, Karolak MJ, lozzo RV, Adams DC, Maridas DE, et al. FOXD1 promotes nephron progenitor differentiation by repressing decorin in the embryonic kidney. Development. 2014;141(1):17-27.

48. Sequeira Lopez ML, Pentz ES, Nomasa T, Smithies O, Gomez RA. Renin cells are precursors for multiple cell types that switch to the renin phenotype when homeostasis is threatened. Dev Cell. 2004;6(5):719-28.

49. Sariola $\mathrm{H}$, Holm K, Henke-Fahle S. Early innervation of the metanephric kidney. Development. 1988;104(4):589-99.

50. James RG, Schultheiss TM. Patterning of the avian intermediate mesoderm by lateral plate and axial tissues. Dev Biol. 2003;253(1):109-24.

51. Lancaster MA, Knoblich JA. Generation of cerebral organoids from human pluripotent stem cells. Nat Protoc. 2014;9(10):2329-40.

52. Hirschi KK. Hemogenic endothelium during development and beyond. Blood. 2012;119(21):4823-7.

53. Medvinsky A, Dzierzak E. Definitive hematopoiesis is autonomously initiated by the AGM region. Cell. 1996;86(6):897-906.

54. Short KM, Combes AN, Lefevre J, Ju AL, Georgas KM, Lamberton T, et al. Global quantification of tissue dynamics in the developing mouse kidney. Dev Cell. 2014;29(2):188-202

55. Wu H, Uchimura K, Donnelly EL, Kirita Y, Morris SA, Humphreys BD. Comparative analysis and refinement of human PSC-derived kidney organoid differentiation with single-cell transcriptomics. Cell Stem Cell. 2018;23(6):869-81.

56. Magella B, Adam M, Potter AS, Venkatasubramanian M, Chetal K, Hay SB, et al. Cross-platform single cell analysis of kidney development shows stromal cells express Gdnf. Developmental biology. 2017.

57. Vivante A, Kohl S, Hwang DY, Dworschak GC, Hildebrandt F. Single-gene causes of congenital anomalies of the kidney and urinary tract (CAKUT) in humans. Pediatr Nephrol. 2014;29(4):695-704.

58. Phipson B, Zappia L, Combes AN. Single cell RNA-Seq of four human kidney orgaoinds. Accession number GSE114802. 2018.

59. Ransick A, Kim AD, De Sena Brandine G, Lindstrom NO, McMahon A. Single Cell RNA-Seq profiling human embryonic kidney cortex cells. Gene Expression Omnibus. Accession number GSE102596. 2018.

Ready to submit your research? Choose BMC and benefit from:

- fast, convenient online submission

- thorough peer review by experienced researchers in your field

- rapid publication on acceptance

- support for research data, including large and complex data types

- gold Open Access which fosters wider collaboration and increased citations

- maximum visibility for your research: over $100 \mathrm{M}$ website views per year

At $\mathrm{BMC}$, research is always in progress.

Learn more biomedcentral.com/submissions 